\title{
Falsa memória e autismo: qual é a extensão dos déficits semânticos?
}

\author{
Cláudia Aparecida Valasek \\ Paulo Sérgio Boggio' \\ Universidade Presbiteriana Mackenzie, São Paulo - SP - Brasil \\ Pedro Albuquerque \\ Universidade do Minho, Braga - Portugal \\ Quirino Cordeiro \\ Faculdade de Ciências Médicas da Santa Casa de São Paulo, São Paulo - SP - Brasil
}

\begin{abstract}
Resumo: Este artigo constitui-se em um estudo de revisão teórica baseado em artigos que abordam o estudo da falsa memória em população com desenvolvimento típico e autismo. Nas últimas décadas, o interesse pela investigação da falsa memória tem se ampliado. No entanto, são observados poucos estudos que investigam os componentes eletrofisiológicos subjacentes envolvidos no reconhecimento verdadeiro e falso, principalmente no que diz respeito a pacientes com autismo. $O$ presente artigo tem como objetivo apresentar os estudos que investigam falsa memória e o processamento semântico em pessoas com desenvolvimento típico por meio do uso da eletroencefalografia, além de apresentar a restrita literatura acerca do tema em pacientes com autismo. Muito se discute sobre déficits semânticos no autismo, mas há ainda poucos estudos nessa área e com resultados contraditórios. Por fim, novas direções para o desenvolvimento e aprimoramento de novos estudos são apresentadas.
\end{abstract}

Palavras-chave: falsa memória; paradigma DRM; autismo; eletroencefalografia; potencial evocado.

\section{FALSE MEMORY AND AUTISM: WHAT IS THE EXTENT OF THE SEMANTIC DEFICITS?}

\begin{abstract}
In the recent decades, the interest in investigation of false memory has been amplified. However, just a few studies investigate the underlying electrophysiological components involved in true and false recognition, especially with regard to patients with autism. The aim of this article is to present the studies investigating false memory and semantic processing in people with typical development using electroencephalography in addition to (presenting) providing the restricted literature on the subject in patients with autism. There is a debate about semantic deficits in autism, but there are few studies in this area and the results are contradictory. Finally, new directions for the development and improvement of new studies are presented.
\end{abstract}

Keywords: false memory; DRM paradigm; autism; electroencephalography; evoked potential.

1 Endereço para correspondência: Paulo Sérgio Boggio, Laboratório de Neurociência Cognitiva e Social e Programa de Pós-Graduação em Distúrbios do Desenvolvimento, Centro de Ciências Biológicas e da Saúde, Universidade Presbiteriana Mackenzie, Rua Piauí, 181, $10^{\circ}$ andar, São Paulo - SP - Brasil. CEP: 01241-001. E-mail: paulo. boggio@mackenzie.br. 
FALSA MEMORIA Y AUTISMO: ¿CUÁL ES LA MAGNITUD DE LOS DÉFICITS SEMÁNTICOS?

\begin{abstract}
Resumen: En las últimas décadas el interés por la investigación de la falsa memoria se ha ampliado. Sin embargo, se observan pocos estudios que investigan los componentes electrofisiológicos subyacentes implicados en el reconocimiento verdadero y el falso, especialmente con respecto a los pacientes con autismo. Este artículo tiene como objetivo presentar los estudios de investigación de la falsa memoria y procesamiento semántico en las personas con desarrollo típico a través del uso de la electroencefalografía, además de presentar la literatura restringida sobre el tema en los pacientes con autismo. Existe un debate acerca de los déficits semánticos en el autismo, pero hay pocos estudios en esta área y con resultados contradictorios. Por último, se presentan nuevas direcciones para el desarrollo y mejora de nuevos estudios.
\end{abstract}

Palabras clave: falsa memoria; paradigma DRM; autismo; electroencefalografía; potenciales evocados.

O interesse crescente no fenômeno das falsas memórias, observado pelo aumento do número de estudos comportamentais e eletrofisiológicos nessa temática, justifica-se pela importância do tema tanto com relação às suas derivações científicas quanto no que concerne à aplicação clínica e prática. Apesar do avanço nos estudos desde a primeira descrição do fenômeno das falsas memórias, pouco se sabe sobre os processos de retenção e recuperação de estímulos/situações em alguns transtornos globais do desenvolvimento. Nos últimos anos, evidências comportamentais e eletrofisiológicas apontam para déficits semânticos em pacientes com transtorno do espectro do autismo que vão além da linguagem. Nesse sentido, o uso de paradigmas de avaliação de falsas memórias pode contribuir para a compreensão das alterações observadas nesse grupo de pacientes.

Este artigo apresenta uma revisão da literatura sobre falsas memórias e autismo, abordando desde os aspectos comportamentais até os eletrofisiológicos. Ao final, apresenta questões em aberto e possíveis direcionamentos de estudos nessa temática. Para esse levantamento, foi utilizada a base de dados Pubmed e Scopus. Os termos utilizados na busca foram: autism, false memories, event related potential (ERP), eletroencepalography, memory, N400, semantic integration. Não foi feita restrição de data, levantando-se assim todos os artigos até 2014. Os critérios para seleção dos artigos foram: 1. artigos originais com dados empíricos relacionados ao tema de falsas memórias que utilizassem o paradigma Deese-Roediger e McDermortt (DRM) como procedimento instrumento de investigação; 2 . artigos com dados eletroencefalográficos, apresentando registros de potenciais evocados em tarefas envolvendo lista de associação de palavras, como o paradigma DRM.

\title{
Falsas memórias
}

As falsas memórias caracterizam-se por distorções da memória verdadeira, uma vez que remetem ao fato de podermos lembrar eventos que na realidade não ocorreram ou que são parcialmente recordados de maneira diferente da situação vivenciada (Roediger \& McDermott, 1995). 
Existem diferentes paradigmas utilizados no estudo de falsa memória. Um dos paradigmas mais utilizados, ao qual daremos enfoque no presente artigo, é o DRM. O desenvolvimento desse paradigma experimental teve início com as pesquisas desenvolvidas por Deese em 1959, que propôs um novo experimento no qual os participantes estudavam uma lista de palavras relacionadas (por exemplo, médico, enfermeira, remédio etc.), em que uma palavra "tema" (ou item crítico), ou seja, que estava diretamente relacionada às demais palavras (por exemplo, hospital), não era apresentada. Posteriormente ao término de memorização das palavras, o pesquisador solicitou que os participantes dissessem que palavras haviam memorizado. Deese observou que os participantes evocavam palavras semanticamente relacionadas com as apresentadas, como a palavra hospital, mesmo que esta não tivesse sido estudada, o que demonstra um tipo de erro denominado intrusão. A partir desse trabalho, anos mais tarde, Roediger e McDermott (1995) readaptaram o procedimento experimental desenvolvido por Deese e cunharam o fenômeno observado de falsa memória. O procedimento de Roediger e McDermott (1995) consistiu na apresentação de listas de palavras (geralmente oito listas compostas por 12 a 15 palavras), e cada palavra estava associada a um item crítico, ou seja, um tema da lista apresentada. No final da apresentação das listas, dois tipos de tarefa podem ser utilizados para avaliar as falsas memórias: tarefas de recordação e de reconhecimento. Na tarefa de evocação, o participante deve lembrar-se espontaneamente das palavras que foram apresentadas. É comum os participantes recordarem uma palavra que possua forte associação semântica, sem que ela tenha sido apresentada anteriormente. Já nas tarefas de reconhecimento, o participante deve selecionar ou identificar um item que já foi apresentado anteriormente. Por exemplo, o experimentador apresenta uma lista de palavras semanticamente relacionadas, como hospital, enfermeira, injeção, remédio etc.

Depois, os participantes devem julgar quais palavras haviam sido vistas anteriormente. Esse teste era composto por

- Palavras-alvo vistas anteriormente, como hospital.

- Palavras que não haviam sido vistas, mas com forte associação semântica, como médico. Esse tipo de palavra foi denominado de item crítico ou distrator crítico por apresentar alto grau de associação semântica.

- Palavras distratoras que não foram apresentadas previamente, mas também são associadas, embora não tão fortemente quanto o item crítico.

- Palavras denominadas de distratores não relacionados que, por sua vez, não haviam sido apresentadas e não têm nenhuma relação semântica com as demais, como trator.

De acordo com Rodrigues e Albuquerque (2007), pode-se observar uma taxa de reconhecimento falso de itens semanticamente relacionados ao item crítico superior a $80 \%$. Diversas variáveis parecem interferir na evocação e no reconhecimento de palavras semanticamente relacionadas. Uma importante variável é a idade. Na literatura, 
poucos estudos nos ajudam a compreender o padrão de produção de falsas memórias em diferentes faixas etárias, ainda mais no que se refere aos experimentos com crianças, em que os resultados encontrados muitas vezes se mostram contraditórios.

A argumentação sobre o aumento das falsas memórias com a idade é suportado por alguns estudos que evidenciaram menor vulnerabilidade das crianças pré-escolares ao efeito DRM, comparativamente às crianças em idade escolar e aos adolescentes (Brainerd, Reyna, \& Forrest, 2006; Howe, 2005; Carneiro, Albuquerque, Fernandez, \& Esteves, 2007). Em contrapartida, estudos como o realizado por Ghetti, Qin e Goodman (2002) levantam a hipótese de que crianças em idade pré-escolar apresentam taxas maiores de evocações falsas quando comparadas aos grupos de crianças escolares e adolescentes. Essas divergências podem ser explicadas por fatores metodológicos, como tempo de apresentação das palavras, tempo de intervalo de retenção e número de associados por lista.

\section{Falsas memórias e autismo}

A produção científica relacionada ao tema é ainda mais restrita quando desejamos investigar de que forma ocorre a produção de falsa memória em populações clínicas, como pacientes com transtornos do espectro do autismo.

O experimento mais conhecido no estudo de falsa memória em pacientes com autismo verificou que a indução de falsa memória por associação semântica é reduzida, ficando evidente um padrão de registro mais literal da informação (Beversdorf et al., 2000). Beversdorf et al. (2000) observaram esse padrão ao investigarem o desempenho de adultos com diagnóstico dentro do espectro do autismo, em comparação a um grupo de adultos sem autismo em tarefa adaptada de DRM. Os autores verificaram melhor discriminação de itens verdadeiros em comparação a itens falsos no grupo com autismo. Se, por um lado, não formar registros de informações não existentes parece uma vantagem; por outro, consolidar informações apenas em sentido mais literal sinaliza possível alteração na integração de elementos distintos por meio de suas associações semânticas, ou seja, uma alteração na configuração e no desenvolvimento de redes semânticas de informação. Em certo sentido, isso culmina em comprometimento na formação de conceitos e na compreensão dos elementos abstratos da linguagem.

Em estudo mais recente, Kamio e Toichi (2007) encontraram alguns resultados semelhantes aos de Beversdorf et al. (2000). O experimento comparou o desempenho de 28 pacientes com transtorno do espectro do autismo, dos quais $13 \mathrm{com}$ alto funcionamento, 15 com síndrome de Asperger (SA) e 15 com desenvolvimento típico. A tarefa consistiu na apresentação auditiva de sentenças e em posterior reconhecimento delas. Os resultados mostraram que pacientes com autismo de alto funcionamento e SA são susceptíveis a ilusões de memória, uma vez que o falso reconhecimento dos itens semanticamente relacionados foi semelhante nos três grupos. Porém, observou-se redução do falso reconhecimento dos itens críticos no grupo com alto funcionamento quando comparado aos outros grupos. Também foi observada uma taxa de reconhe- 
cimento falso semelhante entre os grupos com SA e controle. Os autores sugerem, dessa forma, que pacientes com alto funcionamento apresentam dificuldades para estabelecer relações semânticas. Além disso, justificam que essa menor susceptibilidade a ilusões de memória pode ser derivada da compreensão verbal limitada desses pacientes e de uma dificuldade global de memorização. Assim, apesar de pacientes com autismo de alto funcionamento conseguirem processar informação semântica simples, a baixa capacidade deles na formação de esquemas predomina, o que os torna menos susceptíveis ao falso reconhecimento.

Esses resultados são justificados pelo fato de que pacientes com transtorno do espectro do autismo parecem ter um processamento perceptual que predomina sobre o processamento semântico (Mottron \& Burack, 2001). Por esse motivo, é justificado o aumento da precisão para memória verdadeira, o que levaria pacientes com autismo a ser menos susceptíveis a ilusões de memória.

Resultado controverso ao de Beversdorf et al. (2000) foi encontrado por Bowler, Gardiner e Grice (2000). Ao testarem a susceptibilidade para ilusões de memórias em dez pacientes com SA e adultos com desenvolvimento típico, submetendo os participantes a teste adaptado ao paradigma, com procedimento de julgamento "lembrar/ saber" (paradigma remember/know) (Tulving, 1985), os autores observaram que reconhecimentos verdadeiro e falso não diferiram entre os grupos. Entretanto, a proporção de itens associados recordados foi menor quando comparada ao grupo-controle, indicando assim que as relações semânticas estabelecidas foram menores nos pacientes com SA. Apesar de essas relações ocorrerem em menor proporção, os pacientes estão sujeitos a cometer erros de memórias como pessoas com desenvolvimento típico.

O estudo realizado por Hillier, Campbell, Keillor, Phillips e Beversdorf (2007) investigou as falsas memórias por meio de dois paradigmas: um visual com apresentação de figuras geométricas e um paradigma verbal baseado no método DRM. Foi observado que pacientes com autismo não apresentam desempenho significativamente melhor do que o grupo-controle, exibindo menos falsas memórias e maior reconhecimento de itens que já haviam sido apresentados anteriormente. No paradigma visual, indivíduos com autismo tiveram maior êxito em discriminar itens vistos anteriormente daqueles distratores críticos, exibindo menor número de falsas memórias.

\section{Falsa memória, autismo e eletroencefalografia}

Seguindo esses achados, Valasek (2013) estudou, a partir de um teste baseado no paradigma DRM, o fenômeno das falsas memórias em um grupo de crianças e adolescentes com autismo de alto funcionamento e comparou o desempenho deles ao de um grupo-controle. Porém, em seu estudo, Valasek (2013) utilizou uma técnica que tem sido explorada em diferentes estudos que abordam neurociência cognitiva: a eletroencefalografia.

A eletroencefalografia é uma técnica de mensuração da atividade neuronal que registra a atividade cerebral gerada por populações neurais por meio da captação 
feita por eletrodos fixados no escalpe. Trata-se de uma avaliação não invasiva (Handy, 2005). Para estudos de neurociência cognitiva, interessa analisar o chamado potencial evocado relacionado ao evento (ERP) que se refere à atividade elétrica de determinadas populações de neurônios em resposta à exposição de diferentes modalidades de estímulos/eventos. Os ERPs são compostos por uma série de picos de voltagem que podem ser positivos ou negativos. A nomenclatura usada para a distinção de cada componente refere-se à polaridade (positiva, representada pela letra P, ou negativa, representada pela letra $\mathrm{N}$ ).

Por meio de estudos com ERPs, os pesquisadores têm buscado fontes de déficits em algumas patologias, como no autismo, em que se procura relacionar déficits no processamento sensorial com os comprometimentos em sua cognição social (Jeste \& Nelson, 2009).

Em relação ao uso da eletroencefalografia em estudos que investigam falsa memória em população com desenvolvimento típico, há um componente que tem sido tipicamente observado em tarefas de reconhecimento, o denominado Parietal Old/New Effect. Esse componente é comumente observado em torno de 400-800 ms, com amplitude máxima na região parietal, com maior amplitude para reconhecimento verdadeiro, ou seja, reconhecimento das palavras já estudadas (palavras-alvo) em relação à rejeição correta de palavras associadas semanticamente (falsas pistas) ou distratores (palavras novas sem relação semântica) (Nessler, Mecklinger, \& Penney, 2001; Geng et al., 2007).

Observa-se uma escassez de estudos acerca do tema em crianças e adolescentes com autismo. Um experimento realizado por Valdizán et al. (2003) aproximou-se da temática ao investigar o processamento semântico de informações. Os pesquisadores avaliaram a presença do componente $\mathrm{N} 400 \mathrm{em}$ um grupo com autismo e em um grupo com SA. Para isso, foram apresentados auditivamente 40 pares de palavras, sendo 20 pares congruentes, ou seja, palavras que apresentavam relação semântica entre si (por exemplo, fruta-maçã), e 20 pares de palavras incongruentes que não apresentavam relação entre si (por exemplo, animal-prato). Os resultados mostraram que, apesar de o componente $\mathrm{N} 400$ ser mais negativo em áreas frontocentrais do grupo com autismo quando comparado ao grupo-controle, não foram observadas diferenças para a interação grupo versus tipo semântico, demonstrando assim que o nível semântico assemelha-se nos três grupos. Em relação à latência do componente N400, os autores observaram que o grupo com autismo apresentou maior latência para o componente N400 (em torno de $526 \mathrm{~ms}$ ) quando comparado ao grupo com SA (424 ms) e ao grupo com desenvolvimento típico (409 ms). Dessa forma, ao contrário da hipótese inicial dos pesquisadores, o grupo com autismo apresentou capacidade em discriminar os pares de palavras congruentes e os pares incongruentes, demonstrando assim uma sensibilidade ao contexto semântico.

Retomando o estudo citado anteriormente, a pesquisa de Valasek (2013) foi inovadora na medida em que analisou os componentes eletrofisiológicos durante a realização de tarefa adaptada ao paradigma DRM em um grupo de crianças com autismo, 
fornecendo assim novas contribuições de como ocorrem o processamento e o reconhecimento das informações em pacientes com esse distúrbio do desenvolvimento.

Os autores deste estudo encontraram o componente Parietal Old/New Effect tanto para o grupo de desenvolvimento típico quanto para o grupo de autismo. O componente teve maior amplitude para reconhecimento verdadeiro de itens previamente estudados em comparação a reconhecimento dos itens novos. A observação desse componente em pacientes com autismo desafia hipóteses prévias e sinaliza que eles também possuem sistemas de identificação eletrofisiológicos de itens novos versus antigos. Além disso, foi verificado que componentes precoces no tempo já se diferenciam em função de aspectos semânticos, e a ausência dessa diferenciação nos pacientes com autismo, em uma janela de 200 milissegundos, pode estar na base das alterações comportamentais observadas. Os autores sugerem que novos estudos devem ser conduzidos para melhor descrever o funcionamento semântico desses pacientes, assim como os componentes eletrofisiológicos subjacentes.

Com relação aos resultados comportamentais, não foram observadas diferenças significativas entre os grupos em relação aos acertos por reconhecimentos de palavras-alvo e acertos por rejeição das falsas pistas. No entanto, os pacientes apresentaram desempenho comportamental diferente do grupo-controle em relação aos acertos por rejeição de distratores não relacionados, ou seja, palavras que não estavam presentes na lista original e não tinham qualquer tipo de relação semântica com as previamente apresentadas.

\section{Conclusões}

Dessa forma, como descrito no decorrer do artigo, os estudos que investigam os efeitos de falsa memória no autismo apresentam resultados heterogêneos. Divergências de resultados são observadas principalmente em relação aos dados comportamentais, uma vez que os estudos são voltados, em maior quantidade, para mensuração de acertos e erros, sendo os erros por associação semântica os de maior relevância para os estudos. Além disso, tal fato, em grande parte, é devido a características específicas da amostra, tais como: idade dos participantes, nível intelectual e diferenças de diagnóstico entre os participantes. A diversidade de resultados encontrada pode estar ligada a aspetos metodológicos, tais como: tipo de apresentação das palavras (visual ou auditiva); tempo de apresentação dos estímulos, que podem ser apresentados de forma rápida ou lenta (Gallo \& Roediger, 2002); e tipo de tarefa (evocação ou reconhecimento).

A partir do que foi exposto, a hipótese prévia de que indivíduos com autismo parecem ser menos susceptíveis a ilusões de memória é inconsistente, levando-nos à necessidade de desenvolver novos estudos para melhor investigação desses achados, uma vez que modelos de intervenção vêm sendo estruturados com base na aceitação de que esses pacientes apresentam falhas em suas redes semânticas de informação. Vale ressaltar que as lacunas observadas na compreensão da produção das falsas memórias 
em pessoas com desenvolvimento atípico, como no caso de pacientes com autismo, não se restringem somente a essa população, já que estudos com população típica demonstram-se controversos em alguns aspectos.

Como apresentado anteriormente, uma das medidas que poderão fornecer evidências científicas importantes em relação aos padrões eletrofisiológicos referentes ao armazenamento e à recuperação de memórias verdadeira e falsa é o uso da eletroencefalografia. Porém, a utilização dessa técnica é ainda escassa considerando o número de estudos que investigam as falsas memórias. Isso é ainda mais crítico se olharmos a literatura nacional. A maior parte dos estudos concentra-se em tarefas comportamentais aplicadas em pessoas com desenvolvimento típico. Novos estudos são necessários para investigar os componentes N400 e LPP no que diz respeito à amplitude e à latência desses componentes. Além disso, tarefas que integrem modalidades sensórias variadas também ajudarão a compreender a extensão dos déficits no autismo e a identificar que domínios encontram-se preservados. Isso é crucial para o desenvolvimento de estratégias de intervenção terapêutica baseadas em evidências.

Também se fazem necessários novos estudos que considerem um aprimoramento da metodologia, levando em conta alguns fatores, tais como aumento das amostras e sua divisão em grupos etários, a fim de investigar as possíveis similaridades e/ou diferenças na apresentação dos componentes eletrofisiológicos. Essa diferença no padrão de falsas memórias entre diferentes idades é importante não apenas em população com distúrbios do desenvolvimento, mas também em indivíduos com desenvolvimento típico, uma vez que resultados comportamentais mostram-se controversos. Além disso, será de grande contribuição o desenvolvimento de estudos com diferentes formas de apresentação da tarefa, como a apresentação auditiva dos estímulos no lugar da apresentação visual, o que poderá permitir a aplicação em crianças com idade precoce e em pacientes com outros transtornos do desenvolvimento.

Por fim, o estudo dos mecanismos envolvidos nos processos de produção de memórias falsas pode ajudar no âmbito clínico, como no desenvolvimento de estratégias de intervenção que utilizam como base conteúdos mnemônicos. Mas, para isso, ainda se faz necessária uma melhor caracterização dos déficits semânticos apresentados por essa população.

\section{Referências}

Beversdorf, D. Q., Smith, B. W., Crucian, G. P., Anderson, J. M., Keillor, J. M., Barrett, A. M., Hughes, J. D., Felopulos, G. J., Bauman, M. L., Nadeau, S. E., \& Heilman, K. M. (2000). Increased discrimination of "false memories" in autism spectrum disorder. Proceedings of the National Academy of Sciences, 18(15), 8734-8737.

Bowler, D. M., Gardiner, J. M., Grice, S. P. (2000). Memory illusions: false recall and recognition in adults with Asperger's syndrome. Journal of Abnormal Psychology, 109(4), 663-672. 
Brainerd, C. J., Reyna, V. F., \& Forrest, T. J. (2006). Are young children susceptible to the false-memory illusion? Child Development, 73(5), 1363-1377.

Carneiro, P., Albuquerque, P., Fernandez, A., \& Esteves, F. (2007). Analyzing false memories in children with associative lists specific for their age. Child Development, 78, 1171-1185.

Gallo, D. A., \& Roediger, H. L. (2002). Variability among word lists in eliciting memory illusions: evidence for associative activation and monitoring. Journal of Memory and Language, 47(3), 469-497.

Geng, H., Qi, Y., Li, Y., Fan, S., Wu, Y., \& Zhu, Y. (2007). Neurophysiological correlates of memory illusion in both encoding and retrieval phases. Brain Research, 1136, 154-168.

Ghetti, S., Qin, J., \& Goodman, G. S. (2002). False memories in children and adults: age, distinctiveness, and subjective experience. Developmental Psychology, 38, 705-718.

Handy, T. C. (2005). Event-related potentials: a methods handbook. Cambridge: MIT Press.

Hillier, A., Campbell, H., Keillor, J., Phillips, N., \& Beversdorf, D. Q. (2007). Decreased false memory for visually presented shapes and symbols among adults on the autism spectrum. Journal of Clinical and Experimental Neuropsychology, 29(6), 610-616.

Howe, M. L. (2005). Children (but not adults) can inhibit false memories. Psychological Science, 16(12), 927-931.

Jeste, S. S., \& Nelson. C. A. (2009). Event related potentials in the understanding of Autism Spectrum Disorders: an analytical review. Journal of Autism and Developmental Disorders, 39, 495-510.

Kamio, Y., \& Toichi, M. (2007). Memory illusion in high-functioning autism and Asperger's disorder. Journal of Autism and Developmental Disorders, 37(5), 867-876.

Mottron, L., \& Burack, J. A. (2001). Enhanced perceptual functioning in the development of autism. In J. Burack, T. Charman, P. R. Zelaso \& N. Yirmiya (Eds.). The developmental autism: perspectives from theory and research (pp. 131-148). Mahwah: Erlbaum.

Nessler, D., Mecklinger, A., \& Penney, T. B. (2001). Event related brain potentials and illusory memories: the effects of differential encoding. Cognitive Brain Research, 10(3), 283-301.

Rodrigues, E. P., \& Albuquerque, P. B. (2007). Produção de memórias falsas com lista de associados: análise do efeito do nível de processamento e da natureza da prova de memória. Psicologia USP, 18(4), 113-131. 
Roediger, H. L., \& McDermott, K. B. (1995). Creating false memories: remembering words not presented in lists. Journal of Experimental Psychology: Learning, Memory, and Cognition, 21, 803-814.

Tulving, E. (1985). Memory and consciousness. Canadian Psychology, 26, 1-12,

Valasek, C. A. (2013). Potenciais evocados relacionados à tarefa de Deese, Roediger e McDermott em crianças e adolescentes com transtorno global do desenvolvimento. Dissertação de mestrado, Universidade Presbiteriana Mackenzie, São Paulo, SP, Brasil.

Valdizán, J. R., Abril-Villalba, B., Méndez-García, M., Sans-Capdevila, O., Pablo, M. J., Peralta, P., \& Bernal-Lafuente, M. (2003). Potenciales evocados cognitivos en niños autistas. Revista de Neurologia, 36, 425-428.

Submissão: 4.11.2014

Aceitação: 10.8.2015 